\title{
A NEW METHOD FOR ESTIMATING THE 3D SIZE-DISTRIBUTION- CURVE OF FRAGMENTED ROCKS OUT OF 2D IMAGES
}

\author{
Souhä̈l OUtAL ${ }^{1}$, DOMINIQUe JEULIN² AND JACQues SCHLEIFER ${ }^{2}$ \\ ${ }^{1}$ University of Liège (ULg), GeMMe-MiCa, Sart Tilman B52, Chemin des chevreuils, 1, 4000 Liège, Belgium, \\ ${ }^{2}$ Paris School of Mines, Centre de Morphologie Mathématique, Centre de Géosciences, 35 rue Saint-Honoré, \\ 77300 Fontainebleau, France \\ e-mail: Souhail.Outal@ulg.ac.be \\ (Accepted May 23, 2008)
}

\begin{abstract}
Image analysis of rock fragmentation is used in mines and quarries to control the quality of blasting. Obtained information is the particle-size-distribution curve relating volume-proportions to the sizes of fragments. Calculation by image analysis of this particle-size-distribution is carried out in several steps, and each step has its inherent limitations. We will focus in this paper on one of the most crucial steps: reconstructing the volumes (3D). For the 3D-step, we have noticed that, due to the current acquisition method, there is no correlation between the average grey level of surfaces of the fragments and their third dimension. Consequently volumes (3D) as well as the sizes (1D) has to be calculated indirectly from the extracted projected areas of the visible fragments of images. For this purpose, we have built in laboratory a set of images of fragmented rocks resulting from blasting. Moreover, several tests based on comparisons between image analysis and screening measurements were carried out. A new stereological method, based on the comparison of the densities of probability (histograms) of the same measurements (with very weak covering and overlapping) was elaborated. It allows us to estimate correctly, for a given type of rock, two intrinsic laws weighing the projected areas distribution in order to predict the volumic distribution.
\end{abstract}

Keywords: 3D reconstruction, cumulative passing, histograms, laws of sizes and volumes, projected areas.

\section{INTRODUCTION}

Several systems of measurement of granulometry based on image analysis exists (Hunter et al., 1990; Franklin et al., 1996), however their complete automation and their robustness were never validated (Qian and Hung, 1996). This is due primarily to the problems inherent to the various phases of measurement, which are principally:

- acquisition of images of the fragmented rocks,

- delineation of the fragments present in the image,

- rebuilding the volumic curve out of projected areas (3D aspect),

- and resolution of the errors due to the sampling.

In this work we will focus on the improvement of the 3D aspect. This phase is situated just after the extraction by image analysis of the outlines of the visible fragments in the image. More precisely, it is a question of rebuilding the volumic size-distributioncurve on the basis of the extracted projected areas. However, since the reasoning is made on the projected areas of the fragments, of which the form is very complex, the rebuilding is very problematic. On one side, the usual stereological methods, which are based on sections, do not work. On the other side, no information about the third dimension of the fragments can be extracted from the grey level images. Indeed, no correlation exists between the grey level of the pixels that form the fragments of rocks and their heights.

For the case of rocks fragments, the numerous methods of reconstruction proposed in the literature consist in calculating the volumic size-distributioncurve by using first of all a volumic model based on a shape assumption for the fragments (spheres, cubes, ellipsoids, etc.). The projected areas of the fragments present in the image are thus assumed to be the projection of the volumes representing these shapes. The size and volume allotted to the fragments are thus deduced from the rebuilt shapes. In the second place, since the screening is the reference of the measurement, the granulometric measurements by image analysis are then calibrated with those of the screening by inserting corrective coefficients (Chavez, 1996; Maerz, 1996; Kemeny et al., 1999). In theory, these coefficients must take into account numerous skews (Chavez et al., 1996; Maerz and Zhou, 1999):

- those related to the strong assumptions made on the shape of fragments,

- those related to the errors of the 2D treatments: incorrect delineation of fragments and fusion of fine particles regions, 
- those related to not taking into account the small fragments that fall between and below the bigger ones (segregation),

- and finally, those related to the method of sampling of fragments.

The robustness of these corrective coefficients is generally altered by the interference of all these phenomena. Their stability was indeed demonstrated only for cases of simple forms objects (like spheres).

Thus, in order to gain in robustness, our suggested approach consists in treating exclusively the stereological rebuilding aspect (measurement in a precise way of the size and volume of the fragment starting from its projected area). At this stage, it is question to treat neither the problems related to the covering between fragments, nor to the segregation, nor to the method of sampling.

Moreover, no assumption on shapes will be introduced to model the fragments, but we will rather associate each projected area with a size and with a volume.

More precisely, putting experimentally in correspondence the two histograms of respectively the retained areas and the retained volumes, calculated successively by images analysis and by screening, has allowed us to establish two laws of reconstruction. The first one allows us to connect every projected area to the size of the fragment that it represents, and the second one allows us to connect it to its equivalent volume.

The application of the two laws obtained from this experimental calibration to another case of projected areas representing fragments of the same rock type, for which the cumulated volumic proportions, measured by screening, follow the fitted mathematical model of Rosin-Rammler (Eq. 12), shows that:

- the retained volumes calculated by image analysis and those measured by screening correspond correctly,

- and the parameters of the fitted model are correctly recovered by images analysis.

\section{METHODS}

\section{HISTOGRAMS OF REFUSALS}

Experimentally, in order to avoid the interference of skews related to the image processing, the analyzed fragments are laid out without masking on only one layer, and present their larger areas at the time of acquisition.
The measurement by image analysis and by screening of the same sample of rock fragments provides the histograms of respectively the retained areas and the retained volumes, noted $g_{2 D}\left(r_{2 D}\right)$ and $g_{3 D}\left(r_{3 D}\right)$. Plotted in the same sizes reference mark, these two histograms are not in a good correspondence upon the whole interval representing the classes of sizes (Fig. 1).

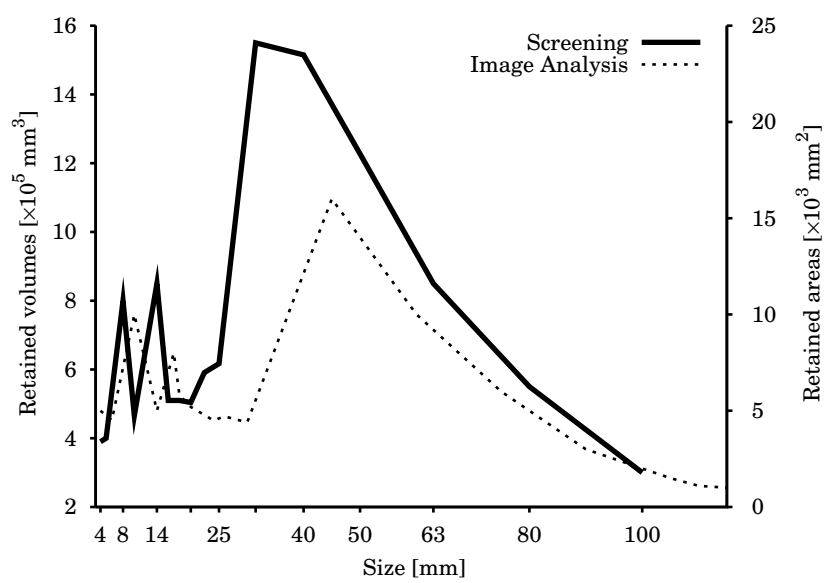

Fig. 1. Histograms of retained areas and retained volumes.

Indeed, two types of shifts can be noted:

- a first weak horizontal shift, where the biggest size estimated by image analysis is higher than the maximum size measured by screening. It is due to the difference of the size concepts of the two measurement systems. Indeed, the screening assigns to the fragment the size of the sieve which retains it (noted $r_{3 D}$ ), whereas for the image analysis, the assigned size is the diameter of the maximum circle registered inside the projected area of the fragment (noted $r_{2 D}$ ).

- The second shift is a vertical shift. It is due to the fact that one compares projected areas with volumes. It expresses, theoretically, the third dimension that connects each projected area to the volume of the fragment that it represents.

The stereological reconstruction which consists of connecting each projected area extracted by image analysis to its corresponding volume measured by screening is thus not directly possible.

It is initially necessary to adjust the classes of sizes of the retained areas histogram with those of the retained volumes histogram. This means redistributing the various projected areas according to the classes of sizes measured by screening. 


\section{ADJUSTMENT OF THE HISTOGRAMS}

The histogram $g_{2 D}$ of the retained areas obtained by image processing can be written in the form:

$$
g_{2 D}\left(r_{2 D}\right)=N_{g r} \bar{A}_{r_{2 D}} f_{2 D}\left(r_{2 D}\right),
$$

when:

- $N_{g r}$ is the total number of the projected areas of fragments;

- $\bar{A}_{r_{2 D}}$ is the average area of the class of size $r_{2 D}$;

- and $f_{2 D}\left(r_{2 D}\right)$ is the number law of the sizes $r_{2 D}$.

The function $\varphi$ which would connect the two sizes $r_{2 D}$ and $r_{3 D}$ would be expressed by the following relation:

$$
r_{3 D}=\varphi\left(r_{2 D}\right)
$$

It results from this:

$$
f_{2 D}\left(r_{2 D}\right)=f_{2 D} \circ \varphi^{-1}\left(r_{3 D}\right) .
$$

The redistribution of the retained areas following the sizes $r_{3 D}$, determined by screening, makes it possible to reformulate the histogram $g_{2 D}$ as follows:

$$
\begin{aligned}
g_{2 D}\left(r_{3 D}\right) & =N_{g r} \bar{A}_{r_{2 D}}\left(f_{2 D} \circ \varphi^{-1}\right)\left(r_{3 D}\right) \\
& =N_{g r} \bar{A}_{r_{2 D}} h\left(r_{3 D}\right)
\end{aligned}
$$

with

$$
h=f_{2 D} \circ \varphi^{-1} .
$$

In practice $\varphi$ must be invertible within the studied interval of the sizes.

However, as the shape of the fragments is very complex and that the reasoning is led on projected areas, it is practically impossible to analytically extract the function $\varphi$.

The possibility offered to us to estimate $\varphi$ is to proceed in an experimental way with the help of the notion of individual classes of sizes.

\section{Individual classes of sizes}

The approach consists in analyzing every individual class of size collectively by both measurement systems. So for every individual class, the fragments were not mixed with those of the other classes. They were rather, at the same time weighed for the measurement of their volumes and acquired by images for the measurement of their projected areas (Fig. 4a,b). In this way, it was possible for us, for the same fragments of a given class, to access collectively to the measure of their sizes and volumes measured by screening $r_{3 D}$ and $V_{3 D}$, as well as to their sizes and projected areas estimated by image analysis $r_{2 D}$ and $A_{2 D}$.

The measurement by screening of the individual class gives place to a size, which is the smallest of the both sieves of the class, and a total volume equal to the sum of the volumes of all the fragments of the class. This measure appears as a single point in the histogram of the retained volumes $\left(r_{3 D}, V_{3 D}\right)$.

The measure by analysis of image of the individual class is different. Indeed, even if it is only about the same class of size, the projected areas of the class fragments do not contain necessarily the same inscribed circle. So the sorting gives place to a measure in the form of a histogram (Fig. 2).

Nevertheless, knowing that it is about the same individual class of sizes, the histogram of the projected areas can be reduced also to a single point. This last one will represent the equivalent size of the class, taken as being the middle of the interval of the sizes of the various projected areas of the class, and a total retained area which is the sum of all the projected areas of the class (Fig. 2).

So the size measured by image analysis will be the sorting size of the individual class $\left(r_{2 D}\right)$.

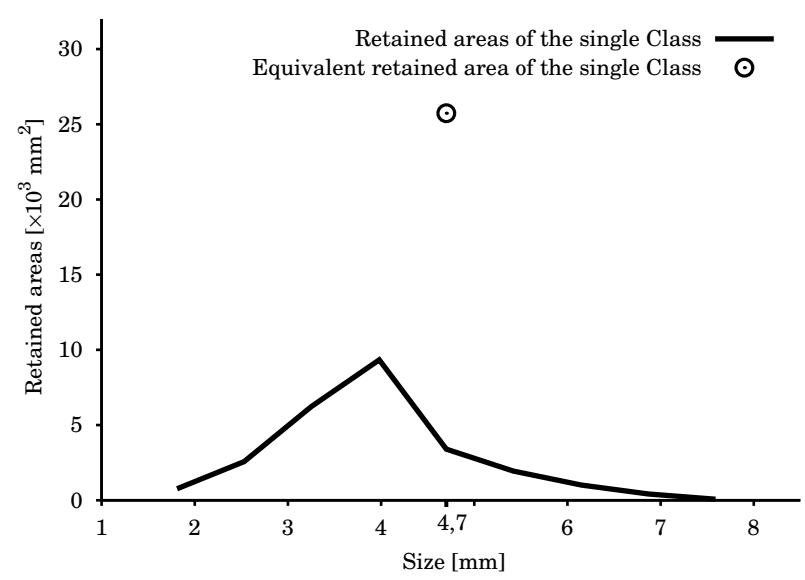

Fig. 2. Histogram of projected areas inside an given individual class.

\section{Mapping of the sizes}

The representation of both measures made on the individual class shows the two shifts previously mentionned (Fig. 3). However, since now we consider individual classes of sizes that we know experimentally, the mapping of both sizes $r_{2 D}$ et $r_{3 D}$ is henceforth possible. 


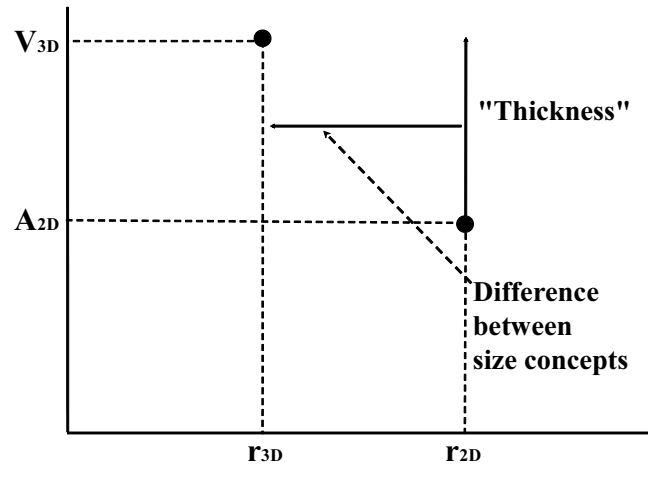

Fig. 3. Shifts between the measures by image analysis and by sieving.

For each individual class of size, the mapping corresponds to the experimental determination of a coefficient $\alpha$ connecting $r_{2 D}$ to $r_{3 D}$ :

$$
r_{3 D}=\alpha r_{2 D}
$$

When one carries out this operation for all of the individual classes of the granulometric distribution, we can define experimentally the function $\varphi$ which expresses the relation between the two sizes.

\section{EXPERIMENTAL LAWS OF RECONSTRUCTION}

Now, by using the total number of fragments of each individual class, we can estimate two experimental laws, which make it possible to rebuild the projected areas. A sizes law and a thicknesses law.

\section{Size law}

The division of the total area (or surface refusal) of each individual class by its total number of the fragments gives the average area equivalent to the class, $\bar{A}_{2 D}$. The size attributed to this average area is the one attributed to the individual class: $r_{2 D}$.

Being given that the relation between $r_{2 D}$ and $r_{3 D}$ is now known, it is also possible to connect experimentally $\bar{A}_{2 D}$ to the size $r_{3 D}$. Lastly, identically, carried within the whole individual classes, this relation defines experimentally a law, which one will name sizes law (Eq. 8). It will make it possible to redistribute the average areas $\bar{A}_{2 D}$ according to the sizes $r_{3 D}$, which is equivalent to a readjustment of the two histograms of the refusals:

$$
r_{3 D}=\tau\left(\bar{A}_{2 D}\right) .
$$

\section{Thicknesses law}

In the same way, the division of the volumic refusal of the class by the total number of the fragments gives the average volume of the class $\bar{V}_{3 D}$. The dimension, which one will name "thickness", connecting the average area $\bar{A}_{2 D}$ to the volume $\bar{V}_{3 D}$ is obtained logically by:

$$
e\left(r_{3 D}\right)=\frac{\bar{V}_{r_{3 D}}}{\bar{A}_{r_{2 D}}} .
$$

Here the index $r_{3 D}$ applied to $\bar{V}$ means the proper volume equivalent to the size $r_{3 D}$ (the notation $\bar{V}_{3 D}$ would like to say here an unspecified volume).

Then, identically to the case of the sizes, the experimental function which expresses, for all the individual classes, the relation between each average area and its equivalent "thickness" defines a law $\eta$, which one will call thicknesses law (Eq. 10). It results from this the calculation of fragments volumes starting from the average areas (Eq. 11).

$$
\begin{aligned}
e\left(r_{3 D}\right) & =\eta\left(\bar{A}_{2 D}\right), \\
\bar{V}_{r_{3 D}} & =\eta\left(\bar{A}_{2 D}\right) \bar{A}_{r_{2 D}} .
\end{aligned}
$$

\section{EXPERIMENTAL MATERIAL}

Two experimental batches of fragments of the same type of rock were prepared by our care in the Center of Geosciences at Paris School of Mines in France (CGES, 2005). It acts of gneiss coming from a blasting at the career de la Clareté of the group CHARIER C.M. (Srhiar, 1999).

\section{Experimental Batch $\mathrm{N}^{0} 1$}

This first batch made up of the individual classes of size was prepared with an aim of establishing experimentally the two laws of rebuilding.

A mass of approximately $19 \mathrm{~kg}$ of fragments with sizes included in the interval $[3.15 \mathrm{~mm}, 63 \mathrm{~mm}$ ] was screened according to the fifteen classes of the interval of sizes. Then the retained volumes of each individual class were weighed.

The fragments contained in each sieve were then spread out without overlaps on a single layer, and without being mixed with those of the other sieves. Fifteen images representing each one all fragments of an individual class were then acquired. Then, the application of automatic delineation algorithms (Outal, 2006) to the acquired grey level images of fragments made it possible to obtain their projected areas (Fig. 4a,b). 


\section{Experimental Batch $\mathrm{N}^{0} 2$}

The second batch, of a mass of approximately $27 \mathrm{~kg}$, was made of other fragments of the same type of rocks as the previous one. The sizes of fragments are also included in the interval $[3.15 \mathrm{~mm}, 63 \mathrm{~mm}]$. For the needs of the validation, the granulometric distribution was prepared in order to fit correctly with the mathematical model of Rosin-Rammler (Eq. 12), also known under the name of Weibull distribution (Patel et al., 1976), with the granulometric parameters: $n=1.48$ and $X c=24.66 \mathrm{~mm}$.

$$
P(x)=100\left(1-\exp \left[-\left(\frac{x}{X_{c}}\right)^{n}\right]\right)[\%]
$$

- $\quad X_{c}$ is the caracteristic size of the distribution; it corresponds to a cumulated passing $P\left(X_{c}\right)$ equal to $63.21[\%]$.

- $\quad n$ is the uniformity index; it provides an indication on the dispersion of the distribution.

This choice of fit will indeed make it possible to test the recovery of the two parameters of the model by image analysis.

Before acquisition, the contents of all the classes were mixed, and as before spread out over only one layer without overlaps. The acquisition of the whole set of fragments gave seven grey level images (mosaic). With the help of the same delineation algorithms, the projected areas of the fragments were then automatically extracted (Fig. 4c,d).

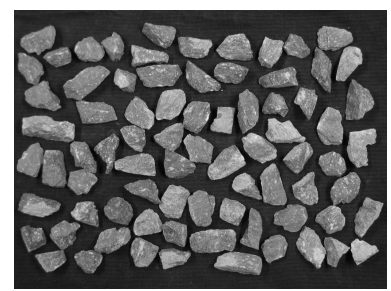

(a)

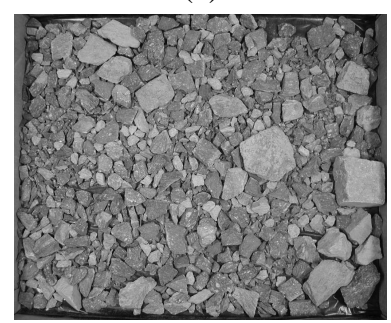

(c)

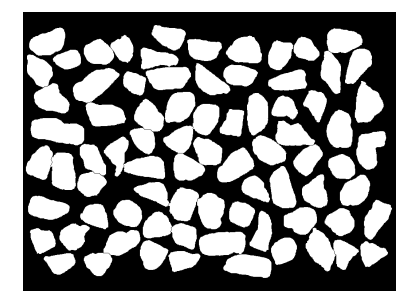

(b)

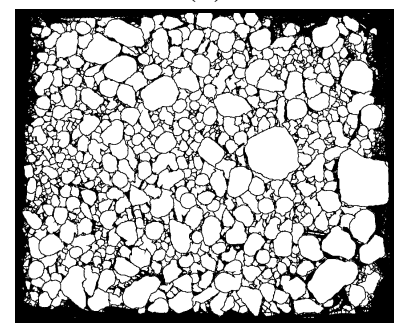

(d)
Fig. 4. (a) Original image from the Batch $N^{o} 1,(b)$ Extracted projected areas, (c) Original image from the Batch $N^{o}$, (d) Extracted projected areas.

\section{RESULTS AND DISCUSSION}

\section{ADJUSTMENT OF THE HISTOGRAMS: PHI LAW}

First of all, the data of respectively the retained areas and the retained volumes of the batch $N^{o} 1$ make it possible to calculate the two histograms $g_{2 D}\left(r_{2 D}\right)$ and $g_{3 D}\left(r_{3 D}\right)$ (Fig. 6).

Each point of the two histograms thus represents the measurement on only one individual class: one image when it acts on $g_{2 D}\left(r_{2 D}\right)$, and one sieve when it acts on $g_{3 D}\left(r_{3 D}\right)$.

As one could expect, the classes of sizes measured by the two systems of measurement do not correspond.

However, the individual classes of screening equivalent to the various images being experimentally known, we can match directly each size $r_{2 D}$ to its equivalent size $r_{3 D}$ (Fig. 5). Then, a least squares fit of these experimental data, provides the function $\varphi(\mathrm{Eq}$. 13).

$$
\begin{aligned}
r_{3 D} & =0.878 r_{2 D} \\
& =\varphi\left(r_{2 D}\right)
\end{aligned}
$$

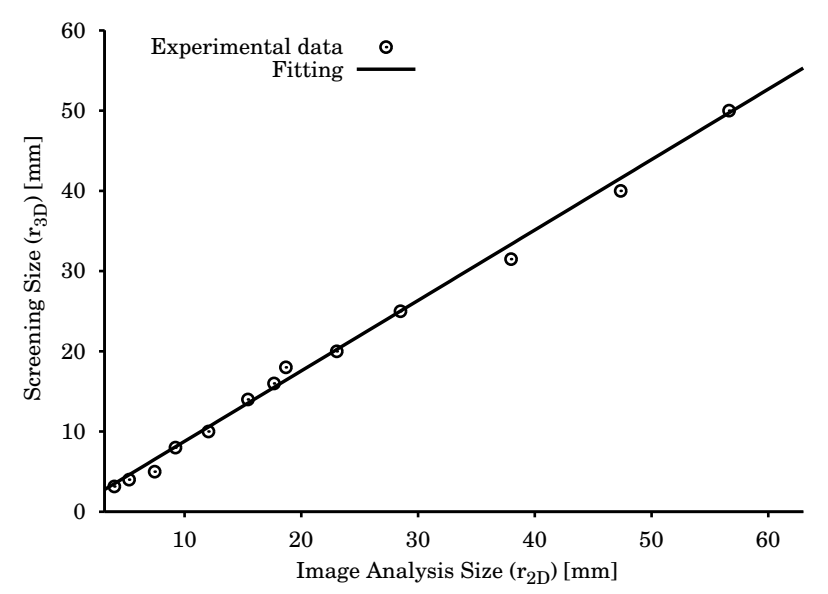

Fig. 5. The $\varphi$ function.

At present, the knowledge of the relation linking both sizes makes it possible to redistribute the retained areas according to sizes $r_{3 D}$, giving access to the histogram $g_{2 D}\left(r_{3 D}\right)$ (Fig. 6).

\section{ESTIMATION OF THE TWO LAWS OF RECONSTRUCTION}

The number of grains of each individual class provided respectively the average projected areas $\bar{A}_{2 D}$ and the average volumes $\bar{V}_{3 D}$ of the various individual classes. 


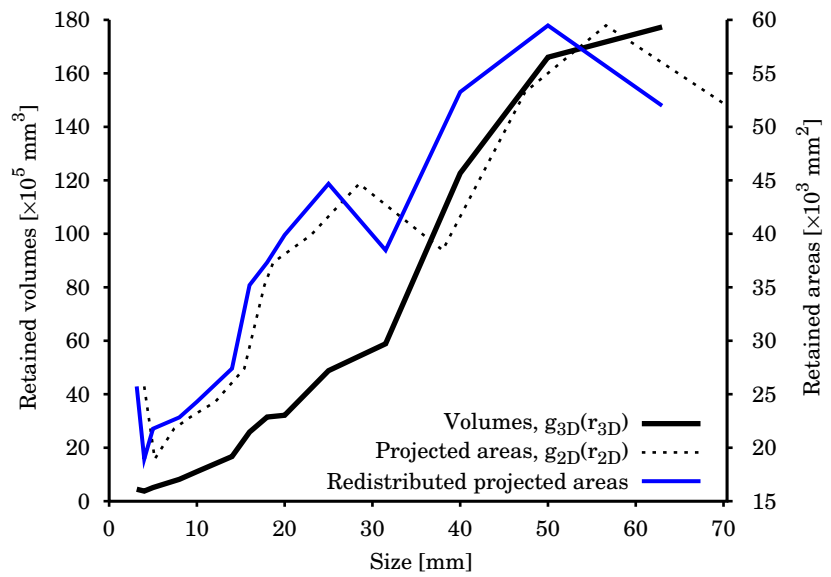

Fig. 6. Redistribution of the classes, obtained by image analysis.

In the same way, the least square fit of the experimental data of the average areas $\bar{A}_{2 D}$, the sizes $r_{3 D}$ and the thicknesses $e\left(r_{3 D}\right)$ makes it possible to obtain the two laws: $\tau$ and $\eta$ (Eqs. 15 and 17; Fig. 7).

$$
\begin{array}{r}
\tau: r_{3 D}=0,63 \bar{A}_{2 D}^{0,5237} \\
\approx 0,7556 \bar{A}_{2 D}^{0,5}, \\
\eta: e\left(r_{3 D}\right)=0,282 \bar{A}_{2 D}^{0,5326} \\
\approx 0,393 \bar{A}_{2 D}^{0,5} .
\end{array}
$$

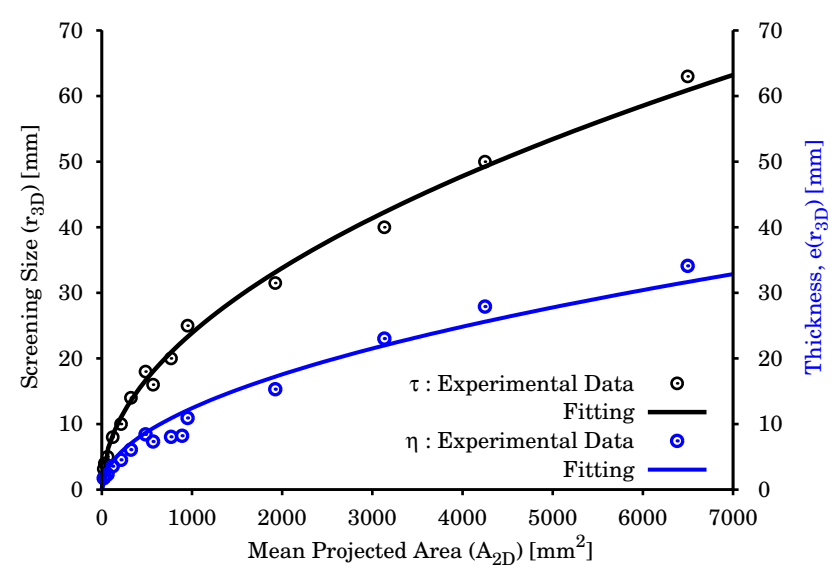

Fig. 7. Experimental laws of reconstruction.

The analysis of the two curves shows on the one hand, that for the two obtained laws, the sizes as well as the thicknesses are proportional to the square root of the average areas. This reveals that although the approach is experimental, the character of homogeneity between handled dimensions is strictly respected. In the other hand, the adjustments carried out show that it is possible to correctly extrapolate the two laws to the large sizes. This enables us in theory to consider their use in the case of distributions which have a maximum size larger than those of the studied sample.

\section{RECONSTRUCTION OF THE PROJECTED AREAS}

The validation of the obtained results, which constitutes the ultimate goal of our study, will concern the rebuilding of the projected areas of the second batch, on the basis of the two laws obtained for the first one. The histogram of the retained volumes estimated by image analysis will thus be compared with the reference measured by screening.

In a second step, the volumic proportions calculated by image analysis will be adjusted with the same fitting model used for the screening. This will make it possible to extract the parameters $n$ and $X_{c}$ of the model, which will be also compared to those of the reference.

The sequence of reconstruction is as follows:

1. Initially, processing the nine images of the batch $N^{o} 2$ provides the histogram of the retained areas of the full distribution, $g_{2 D}\left(r_{2 D}\right)$ (Fig. 8).

2. For each class of size, the counting of the grains gives the average areas $\bar{A}_{2 D}$. Then the two laws $\tau$ and $\eta$, obtained for the batch $N^{o} 1$, make it possible to assign to each average area its size $r_{3 D}$, and its average volume $\bar{V}_{3 D}$. The figure 8 presents the two histograms of the retained areas and the retained volumes. This figure relates to only the data of the image processing. One can notice, on this level, that the volumes calculated by image analysis are already distributed according to the sizes $r_{3 D}$. Nevertheless, if one considers the classes of the usual sieves, all occurs as if they were sorted more finely according to more sieves.

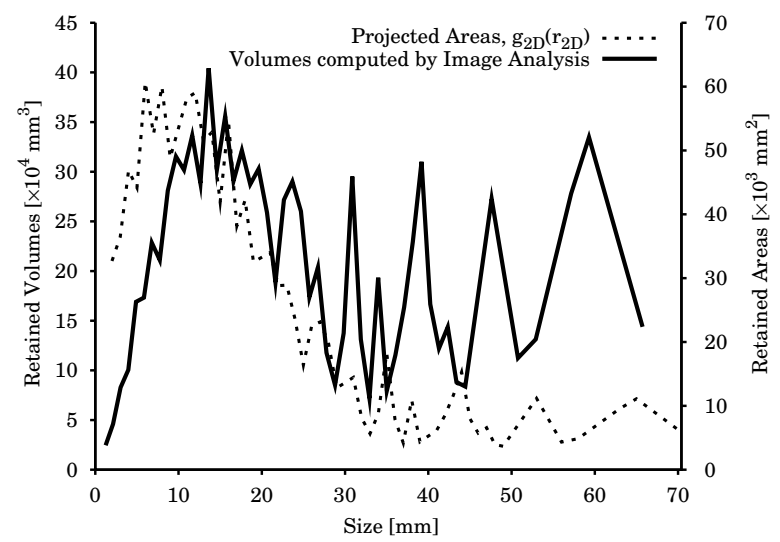

Fig. 8. Retained areas and retained volumes computed by image analysis.

3. Thus, to be able to compare the results of the two techniques of measurement, the retained volumes estimated by image analysis must be regrouped according to the usual classes of the screening. The 
regrouping is carried out by adding the retained volumes, calculated by image analysis, which are in intermediate sizes classes included in the usual classes of sieves (Fig. 9). The new histogram of the volumes calculated by image analysis corresponding to the regrouping is presented in Fig. 10.

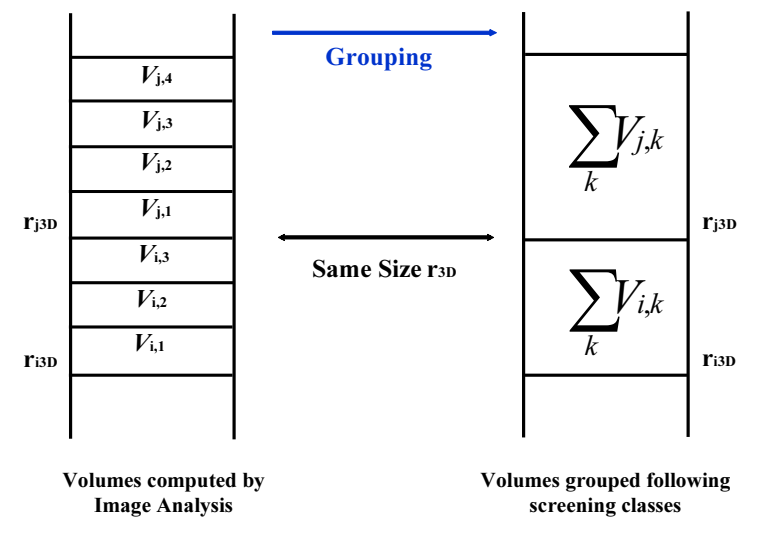

Fig. 9. Diagram of the retained volumes regrouping.

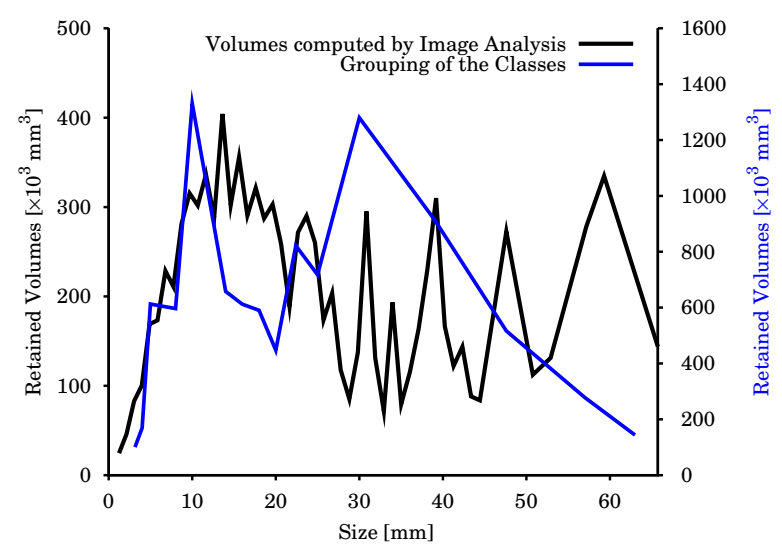

Fig. 10. Regrouping the retained volumes following the usual sieves.

4. Finally, the histogram of the retained volumes estimated by image analysis is compared to the reference measured by screening (Fig. 11).

This last figure shows that the retained volumes were correctly calculated by image analysis, so that the two histograms follow the same trends on the full interval of sizes. In addition, the total volumes obtained by the two systems of measurement are almost identical. In other respects, two types of differences between the data of the two histograms can be noted:

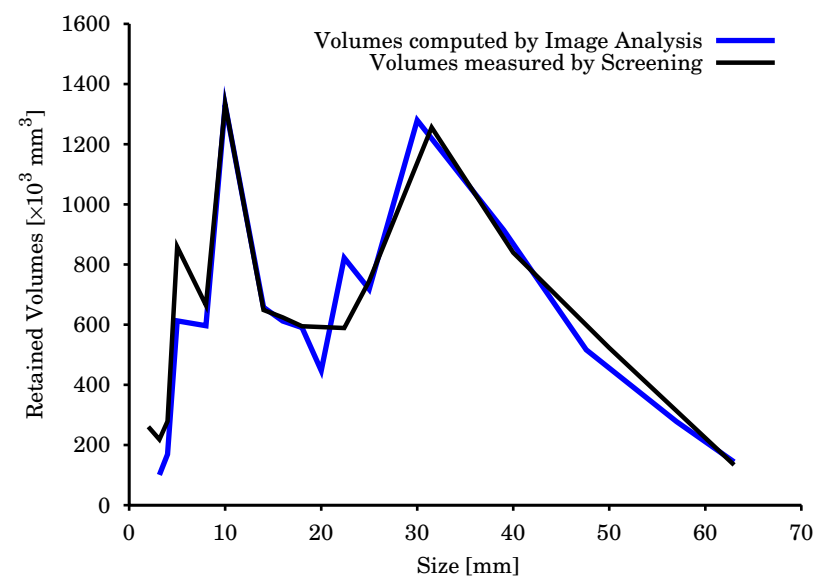

Fig. 11. Retained volumes according to image analysis and screening.

- the first variations are noted for the retained volumes of the classes of small sizes. They can be explained by the fact that some over-segmentations and fusions of the small particles, due to image processing, deteriorate the distribution of surfaces within the classes of small sizes. This inevitably gives place to different volumes from those of the reference for these classes. However, surfaces brought into play (and as well thicknesses) being small, this has no large incidence on the total volume of the distribution.

- The second variations are noticed for the large sizes, where retained volumes are equal but are allotted to slightly different sizes. These shifts are due to the fact that the sizes and the thicknesses of the large fragments calculated by adjustment differ slightly from those of the reference. The quantity of fragments, used at the time of calculations of the two distributions, is thus not representative enough to be able to avoid these small variations. These variations can be reduced by increasing the amount of fragments analyzed for these classes, during the estimation of the two laws of reconstruction.

\section{CUMULATIVE PASSING}

The volumic proportions of the batch $N^{o} 2$ were calculated starting from the retained volumes estimated by analysis of images. Then, the data obtained were fitted to the same model and were plotted in the same reference mark of size as for the reference volumic proportions (Fig. 12). 


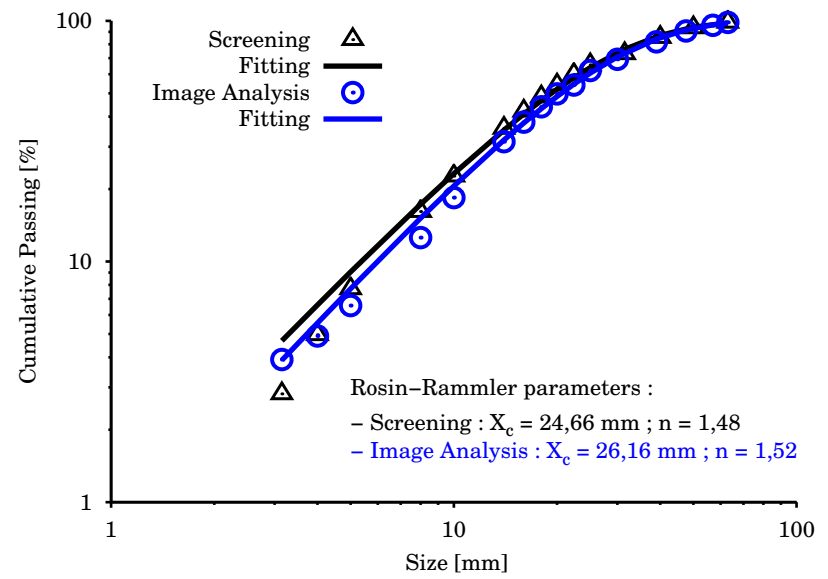

Fig. 12. Volumic proportions measured by image analysis and by screening.

The figure shows that the volumic proportions established by the two measurement techniques are very close for all the sizes classes. Moreover, the curves of the fit to the Rosin-Rammler model are practically merged, result that we expected since the histograms of the refusal are almost equal. It also results from this fact that the orders of magnitude of the parameter of uniformity $n$ and the characteristic size $X_{c}$, computed starting from the curves fit, are correctly recovered by image analysis.

\section{CONCLUSION}

The aim of this work was to develop a new simple and precise method of reconstruction of the volumic size-distribution-curve of the fragmented rocks starting from image analysis. The approach was related particularly to the stage of the stereological reconstruction. The major problem of fitting the two histograms of the retained areas and the retained volumes was solved using the analysis of individual classes of sizes obtained jointly by the two measurement systems. This has led us to develop the two experimental laws connecting intrinsically each projected area to its size and its volume. The robustness of the method was validated through the analysis carried out on the two studied batches.

Two essential points distinguish the approach from the usual methods:

- on the one hand, no assumption on the shape of the fragments was made. Thus, by proceeding experimentally, the correct assignment of the sizes to projected areas made it possible to correctly estimate the spreading out of the size-distributioncurve by image analysis. In the same way the correct rebuilding of volumes provided volumic refusals practically equal to those of the reference.
- on the other hand, in order to separate the eventual problems related to sampling from the image processing aspects, the method was based at the begining on the results of the screening, and the reconstruction was carried out on images of non overlapping fragments. So, the final statistical corrections to correct skews related to the problems of sampling are correctly controlled.

In addition, except for the computation of the volumic cumulated proportions which is the principal objective of granulometric measurement, the approach is of a great industrial interest since it makes it possible to estimate material refusals. In particular, masses of the contents of the various classes, with the help of the consideration of the analyzed rocks densities. This was not available from former methods which estimate relative volumes.

In the same direction, the accessibility of the correct masses of the various classes will also make it possible to consider the insertion of the method in a theory of sampling, such as P. Gy's sampling theory (Gy, 1975).

Let us note also that we do not have an obligation of fitting the experimental volumic proportions to mathematical models since the reasoning is led on refusal.

Lastly, it should be claimed that the two laws of rebuilding are extrapolated correctly on the level of the large sizes for the type of analyzed rocks. If it is proved that this property remains valid for other types of rocks fragmented under different conditions, the estimation of the two laws on a reduced sample will enable us to consider their application to more spread out distributions. This will free us to have to handle very large sizes fragments.

\section{ACKNOWLEDGMENTS}

This research was worked out when Souhail Outal was a PhD student within the Center of Geosciences at Paris school of Mines, France. The authors, and more specially Souhail Outal, would like to thank Serge Beucher and Bruno Tessier (ENSMP, France), and Eric Pirard (University of Liège, Belgium) for their helpful comments and suggestions.

\section{REFERENCES}

Chavez R (1996). Mise au point d'outils pour le contrôle du tir à l'explosif sous contraintes de production. $\mathrm{PhD}$ thesis. École des Mines de Paris.

Chavez R, Cheimanoff N, Schleifer J (1996). Sampling problems during grain-size distribution measurements. Rock Fragmentation by Blasting. In: Proc Congr Fragblast 5. 
Gy P (1975). Théorie et pratique de l'échantillonnage des matières morcelées. Cannes: ED.PG.

Hunter GC, McDermott C, Miles NJ, Singh A, Scoble MJ (1990). A review of image analysis techniques for measuring blast fragmentation. Mining Science and Technology 11:19-36.

Franklin JA, Kemeny JM, Girdner KK (1996). Evolution of measuring systems: A review. In: Franklin J, Katsabanis T, eds. Measurement of Blast Fragmentation. Rotterdam: Balkema, 47-52.

Kemeny JM, Girdner KK, Bobo T, Norton B (1999). Improvements for fragmentation measurement by digital imaging: Accurate estimation of fines. In: Proc 6th Int Symp Rock Fragment Blast, 103-9.

Maerz NH (1996). Reconstructing 3-d block size distribution from 2-d measurements. In: Franklin J, Katsabanis T, eds. Measurement of Blast Fragmentation. Rotterdam: Balkema, 39-43.

Maerz NH, Zhou W (1999). Calibration of optical digital fragmentation measuring systems. Johannesburg, South African Institute of Mining and Metallurgy. Fragblast 1999:125-31.

Outal S (2006). Quantification par analyse d'images de la granulométrie des roches fragmentées: amélioration de l'extraction morphologique des surfaces, amélioration de la reconstruction stéréologique. $\mathrm{PhD}$ thesis, École des Mines de Paris.

Patel JK, Kapadia CH, Owen DB (1976). Handbook of statistical distributions. New York: Marcel Dekker, 1976.

Qian L, Hung T (1996). Comparing systems - Validation of FragScan, WipFrag and Split. In: Franklin J, Katsabanis $\mathrm{T}$, eds. Measurement of Blast Fragmentation. Rotterdam: Balkema, 151-5.

Srhiar T (1999). Étude et amélioration du processus de mesure de la granularité par analyse d'images: Réalisation d'un bans d'essais. PhD thesis, École des Mines de Paris. 